\title{
Theoretical Investigation of the Displacement Burst Observed in Nanoindentation by Collective Dislocation Loops Nucleation Model ${ }^{*}$
}

\author{
Tomohito TSURU $^{* *}$ and Yoji SHIBUTANI ${ }^{* * *}$ \\ **Nuclear Science and Engineering Directorate, Japan Atomic Energy Agency, \\ 2-4 Shirakata-Shirane, Tokai-mura, Ibaraki 319-1195, Japan \\ E-mail: tsuru.tomohito@jaea.go.jp \\ ${ }^{* * *}$ Department of Mechanical Engineering, Graduate School of Engineering, Osaka University, \\ 2-1 Yamada-oka, Suita, Osaka, 565-0871, Japan \\ E-mail: sibutani@mech.eng.osaka-u.ac.jp
}

\begin{abstract}
Abrupt growth of displacement observed in the relationship between indent load and indent depth in nanoindentation of crystalline materials, so-called displacement burst, has been recognized as one of the representative examples of nanoscale plastic behavior (nanoplasticity). This phenomenon corresponds to the early stage of plastic deformation and is greatly influenced by the collective dislocation emission. In the present paper a simplified model is constructed for the first displacement burst with use of the elastic theory based on both the Hertzian contact theory and the classical dislocation theory to evaluate the displacement burst in nanoindentation. The result of the analytical model for the energy equilibrium revealed that there is a strong correlation between burst width and critical indent depth that corresponds to the dislocation emission. Finally, it is shown that more than one hundred high-density dislocations are generated simultaneously and surface step corresponding to the Burgers vector of dislocation dipole of each emitted dislocation causes significant displacement burst.
\end{abstract}

Key words: Nanoindentation, Displacement Burst, Collective Dislocation Emission, Elastic Theory of Dislocations

\section{Introduction}

Recent advances in material miniaturization and highly accurate measurement techniques have allowed the mechanical properties of nanoscale materials to be measured. Nanoindentation has been widely used because of its applicability in ambient conditions. An abrupt increase in indent depth that occurs subsequent to homogeneous elastic deformation, or "displacement burst", can be observed in metallic materials below the 100-nm scale of the indent depth ${ }^{(1)-(3)}$. This anomalous behavior is thought to be caused by the collective dislocation motion specific to nanoscale plastic deformation (Nanoplasticity) due to the characteristics of crystal orientation dependence ${ }^{(4)}$. High-density dislocation groups can actually be observed with in-situ nanoindentation using Transmission Electron Microscopy (TEM) ${ }^{(5)}$. In spite of a large number of observations for the burst behavior, however, the evolution of dislocation structures with nanoindentation and its relationship to mesoscopic burst behavior remain controversial issues. Recently indentation analysis via atomistic simulation such as Molecular Dynamics (MD) has been widely performed because of the

${ }^{*}$ Received 24 Sep., 2008 (No. T1-06-1175) Japanese Original : Trans. Jpn. Soc. Mech. Eng., Vol.73, No.732, A (2007), pp.877-882 (Received 1 Dec., 2006) [DOI: 10.1299/jcst.2.559] 
development of high performance computing. The authors have reported that the initial dislocation emission requires much higher critical shear stress than the theoretical shear strength, and that Prismatic Dislocation Loops (PDL) are formed by both the unstable interaction between dislocations and cross slip ${ }^{(6),(7)}$. Nevertheless, a large gap exists between atomic scale simulations and actual experiments, and the relationship between the collective dislocation motion and experimentally-observed anomalous behavior is yet to be fully understood.

In the present paper, particular note is taken of the deformation properties up to the first burst. In this stage the indentation profile can be divided into the purely elastic deformation before the burst and the irreversible dislocation nucleation during the burst. Hence a simplified analytical model based on the energy equilibrium was constructed. That is, the elastic strain energy stored by indentations evaluated using Hertzian contact mechanics is converted into the total energy of dislocations, which is composed of the self energy and the interaction energy between dislocations. The physical mechanism of the displacement burst is then discussed using the new analytical model.

\section{Elastic strain energy stored by nanoindentation}

It is known from previous studies that a considerably high compressive pressure is generated causing an elevation in the critical shear stress for dislocation emissions ${ }^{(6),(7)}$. In contrast to the consideration of local stress, the energy equilibrium of the system in nanoindentation is noted. A pattern diagram of the experimentally observed indent load depth curve is given in Fig 1. This curve is divided into the processes of elastic and plastic deformations. An ideal analytical model was then constructed, in which the elastic strain energy stored by the indentation evaluated using Hertzian contact mechanics is converted into the total dislocation energy. This model can be used for the deformation properties up to the first burst as it is based on elastic theory and dislocation characteristics differ with the material $^{(4)}$.

The stored strain energy is equal to the work done by the indenter, equivalent to the hatched area in Fig 1, and can be calculated using the following equation:

$$
W_{\mathrm{E}}=\int_{0}^{h_{\mathrm{c}}} P(R, h) d h,
$$

where $P$ is the indent load, $R$ the radius of the indenter tip and $h$ the indent depth. And although the indentation test in the present work was performed using a Berkovich type indenter, the indenter tip can also be approximated with a spherical indenter in the case of small indent load up to at least the first burst. Hence load $P(R, h)$ is evaluated using the Hertzian solution ${ }^{(8)}$ and provides the following relationship:

$$
P(R, h)=\frac{4}{3} E^{*} R^{1 / 2} h^{3 / 2},
$$

where $E^{*}$ is the reduced elastic modulus for indentation which is expressed by Young's modulus and the Poisson ratio of both the indenter (expressed as $i$ ) and the specimen (expressed as $s$ ).

$$
\frac{1}{E^{*}}=\frac{1-v_{\mathrm{s}}^{2}}{E_{\mathrm{s}}}+\frac{1-v_{\mathrm{i}}^{2}}{E_{\mathrm{i}}} .
$$

Because of the much larger Young's modulus for a diamond indenter than a metal specimen, the approximation, $E_{\mathrm{i}} \rightarrow \infty$, can be considered appropriate in Eq. (3). When Young's moduli and the Poisson ratios for $\mathrm{Al}$ and $\mathrm{Cu}$ are referred to in Smithells's database ${ }^{(9)}$, the reduced moluli $E^{*}$ are 80.1 and $147.1 \mathrm{GPa}$ for $\mathrm{Al}$ and $\mathrm{Cu}$, respectively. Hence the elastic strain energy can be evaluated as follows:

$$
W_{\mathrm{E}}=\frac{8}{15} E^{*} R^{1 / 2} h_{c}^{5 / 2} .
$$




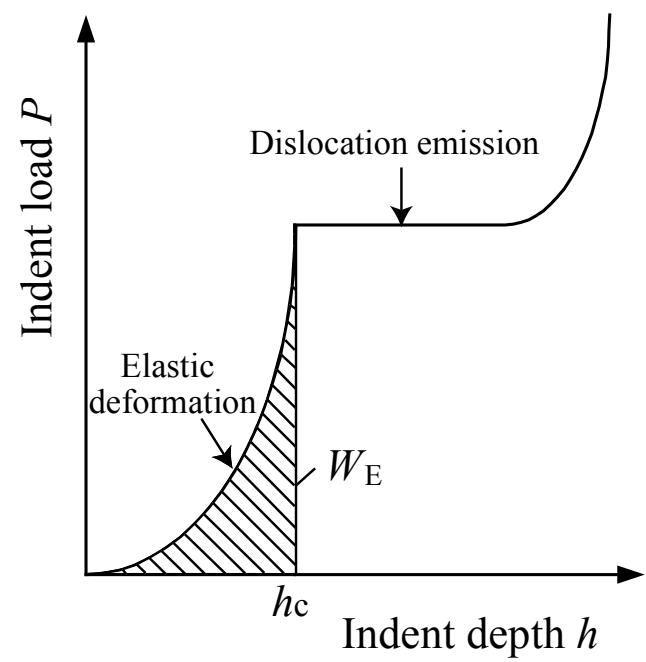

Fig. 1 Diagram of elastic deformation and dislocation emission in the relationship between load and depth.

\section{Interaction energy of dislocations}

When dislocations are present in the infinite field of a material the total dislocation energy can be expressed as follows:

$$
W_{\mathrm{D}}=\sum_{\alpha<\beta} W_{\alpha \beta}+\sum_{\alpha} W_{\mathrm{self}}+\sum_{A} \gamma_{\mathrm{SF}}
$$

where $W_{\alpha \beta}$ is the interaction energy between dislocations, $W_{\text {self }}$ the self energy of a dislocation, and $\gamma_{\mathrm{SF}}$ the stacking fault energy. It was discovered in atomistic simulations carried out by the authors that shear dislocation loops on the $\{111\}$ plane create Prismatic Dislocation Loops (PDL) which move in the $<110>$ direction. Two circular loops with the same radius and the same Burgers vector normal to the loop plane are considered here. A diagram of the system for two dislocations is given in Fig 2, with the interaction energy of the system being provided by the following equation ${ }^{(10)}$ :

$$
\begin{aligned}
W_{12}= & -\frac{\mu}{2 \pi} \oint_{C_{1}} \oint_{C_{2}} \frac{\left(\boldsymbol{b}_{1} \times \boldsymbol{b}_{2}\right) \cdot\left(d \boldsymbol{l}_{1} \times d \boldsymbol{l}_{2}\right)}{R}+\frac{\mu}{4 \pi} \oint_{C_{1}} \oint_{C_{2}} \frac{\left(\boldsymbol{b}_{1} \cdot d \boldsymbol{l}_{1}\right)\left(\boldsymbol{b}_{2} \cdot d \boldsymbol{l}_{2}\right)}{R} \\
& +\frac{\mu}{4 \pi(1-v)} \oint_{C_{1}} \oint_{C_{2}}\left(\boldsymbol{b}_{1} \times d \boldsymbol{l}_{1}\right) \cdot \boldsymbol{T} \cdot\left(\boldsymbol{b}_{2} \times d \boldsymbol{l}_{2}\right)
\end{aligned},
$$

where $\mu$ is the shear modulus, $v$ the Poisson ratio, and $T_{i j}=\partial R / \partial x_{i} \partial x_{j}$. In this equation both $\boldsymbol{b}_{1} \times \boldsymbol{b}_{2}=\mathbf{0}$ and $\boldsymbol{b}_{1} \cdot d \boldsymbol{l}_{1}=\boldsymbol{b}_{2} \cdot d \boldsymbol{l}_{2}=\mathbf{0}$ due to the crystallographic direction of the PDL, and therefore only the third term contributes to the integration. And the differentiations of

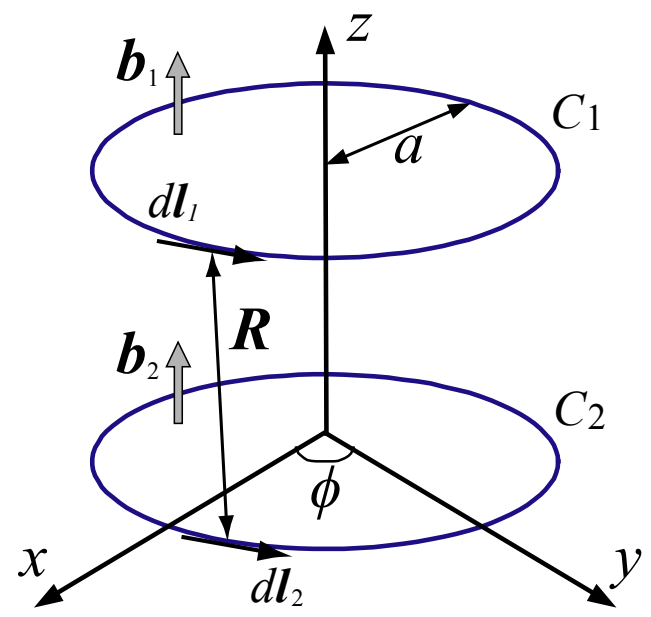

Fig. 2 Interaction between two circular loops with the Burgers vector perpendicular to loop plane. 
$T_{i j}$ in terms of $R$ and $z$ are equal to zero since $\left(\boldsymbol{b}_{1} \times d \boldsymbol{l}_{1}\right)$ and $\left(\boldsymbol{b}_{2} \times d \boldsymbol{l}_{2}\right)$ are perpendicular to the $z$ direction. In addition, upon taking the infinitesimal linear element $d \boldsymbol{l}_{1}$ parallel to the $y$ direction into consideration, and performing a curvilinear integral along $C_{2}$ Eq. (6) results in Eq. (7) because of the symmetrical property of $C_{1}$.

$$
\begin{aligned}
W_{12} & =\frac{\mu}{4 \pi(1-v)} \int_{0}^{2 \pi}\left\{a^{2} b^{2}\left(\frac{d^{2} R}{d x^{2}} \cos \phi d \phi d \theta+\frac{d^{2} R}{d x d y} \sin \phi d \phi d \theta\right)\right\} \\
& =\frac{\mu b^{2}}{4 \pi(1-v)} 2 \pi a^{2} \int_{0}^{2 \pi}\left\{\frac{1}{R} \cos \phi-\frac{a^{2}(1-\cos \phi)^{2}}{R^{3}} \cos \phi+\frac{a^{2}(1-\cos \phi) \sin \phi}{R^{3}} \sin \phi\right\} d \phi
\end{aligned}
$$

As a result of the variable transformation Eq. (7) becomes a complete elliptic integral. Substituting $d_{\mathrm{D}}$ into $z$ results in the following equations for the cases of $d_{D} \ll a$ and $d_{D} \gg a$, respectively ${ }^{(11),(12)}$.

$$
W_{12}=\frac{\mu b^{2}}{1-v} a\left(\ln \frac{8 a}{d_{\mathrm{D}}}-1\right),\left(\text { in } d_{D} \ll a\right), \quad W_{12}=\frac{2 \pi \mu b^{2}}{1-v} a\left(\frac{a}{d_{\mathrm{D}}}\right)^{3},\left(\text { in } d_{D} \gg a\right) \text {. }
$$

The self energy of each dislocation loop can be obtained using Eq. (8) when considering $d_{\mathrm{D}}$ $=\rho(\rho$ : radius of dislocation core $)$ and then divided by two, as follows ${ }^{(13)}$ :

$$
W_{\text {self }}=\frac{\mu b^{2}}{2(1-v)} a\left(\ln \frac{8 a}{\rho}-1\right) \text {. }
$$

With use of Eq. (8) and (9), the total energy of the dislocations given in Fig 3, where $N$ dislocations with the same radius $a$ on the same slip system align at regular intervals, can be evaluated with the following equations:

$$
\begin{aligned}
W_{\mathrm{D}} & =n_{\mathrm{s}} \frac{\mu b^{2}}{1-v} a\left\{\frac{N}{2}\left(\ln \frac{8 a}{\rho}-1\right)+\frac{N(N-1)}{2}\left(\ln \frac{8 a}{d_{\mathrm{D}}}-1\right)-\sum_{\alpha}^{N-1}(N-\alpha) \ln \alpha\right\} . \\
& +2 \pi a \omega_{\mathrm{SF}} \gamma_{\mathrm{SF}} \cdot n_{s} N,\left(\text { in } d_{D} \ll a\right) \\
W_{\mathrm{D}} & =n_{\mathrm{s}} \frac{\mu b^{2}}{1-v} a\left\{\frac{N}{2}\left(\ln \frac{8 a}{\rho}-1\right)+2 \pi\left(\frac{a}{d_{\mathrm{D}}}\right)^{3} \sum_{\alpha=1}^{N-1} \frac{N-\alpha}{\alpha^{3}}\right\} . \\
& +2 \pi a \omega_{\mathrm{SF}} \gamma_{\mathrm{SF}} \cdot n_{s} N,\left(\text { in } d_{D} \gg a\right)
\end{aligned}
$$

Here $n_{\mathrm{s}}$ is the number of the activated slip system. Previous atomistic simulations indicated that the activated slip system depends on the indentation plane. Eight slip systems are activated and four PDL directions presented when the indentation is implemented on the (001) plane. Therefore $n_{\mathrm{s}}=4$ is derived, and similarly $n_{\mathrm{s}}=2$ on the (110) and $n_{\mathrm{s}}=3$ on the

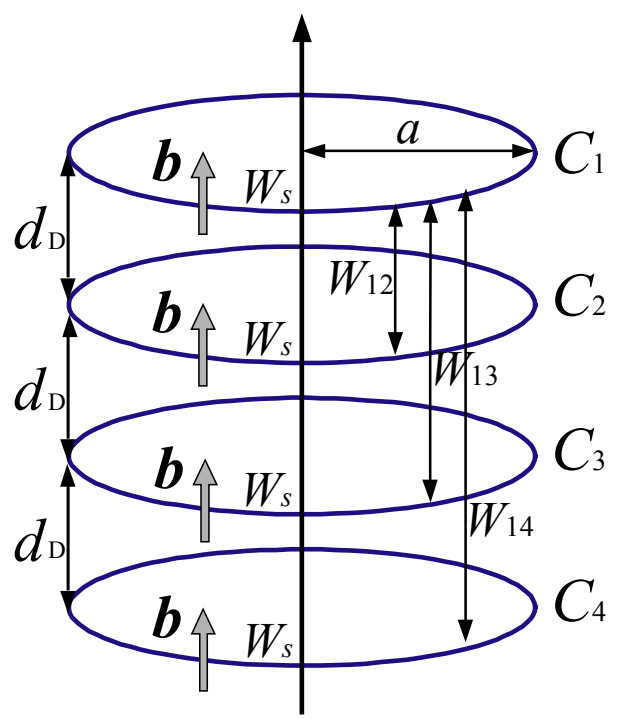

Fig. 3 Interaction between ranged circular dislocation loops of distance $\mathrm{dD}$ between loops. 
(111). Because the Burgers vector for the PDL is normal to the loop plane, the area of the stacking fault is given by $2 \pi a \omega_{\mathrm{SF}}$. The mechanical properties of $\mathrm{Al}$ and $\mathrm{Cu}$ for use in Eq. (10) and (11) are summarized in Table 1. All the properties were calculated with the EAM type interatomic potential proposed by Mishin et al. ${ }^{(14),(15)}$ except for the Poisson ratio. The Poisson ratio was referred to in the Smithells database ${ }^{(9)}$. Additionally $\{111\}\langle 11 \overline{2}\rangle$ Shear modulus $\mu$ and stacking fault energy $\gamma_{\mathrm{SF}}$ have previously been reported by the authors ${ }^{(16)}$.

Although the shape of the PDL emitted with nanoindentation is rhombic, the model used the assumption that circular PDLs are nucleated, and therefore the validity of the circular loop should be verified. The stress distributions of both square and circular dislocations are calculated as shown in Fig 4, using the basis of the energy of dislocations within the frame-

Table 1 Mechanical properties of $\mathrm{Al}$ and $\mathrm{Cu}$ for evaluation of total dislocation energy.

\begin{tabular}{ccc}
\hline Properties & $\mathrm{Al}$ & $\mathrm{Cu}$ \\
\hline \hline$\{111\}\langle 11 \overline{2}\rangle$ Shear modulus $\mu[\mathrm{GPa}]$ & 27.9 & 41.1 \\
Poisson ratio $\nu^{(9)}$ & 0.345 & 0.343 \\
Stacking fault energy $\gamma_{\mathrm{SF}}\left[\mathrm{mJ} / \mathrm{m}^{2}\right]$ & 156.6 & 44.7 \\
Width of stacking fault $\omega_{\mathrm{SF}}[\mathrm{nm}]$ & 0.143 & 3.834 \\
Magnitude of Burgers vector $b[\mathrm{~nm}]$ & 0.286 & 0.256 \\
\hline
\end{tabular}

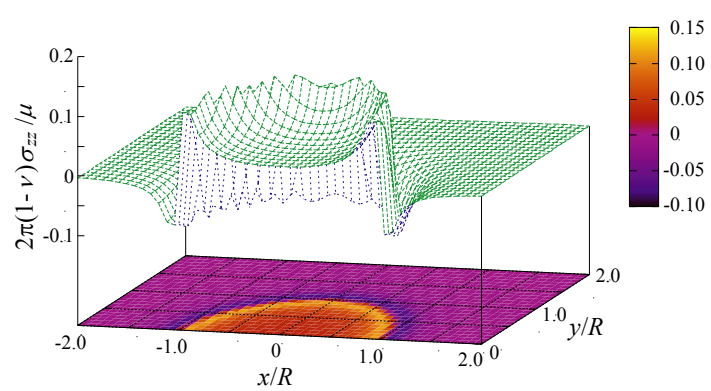

(a) Stress distribution of circular loop on $x y$-plane

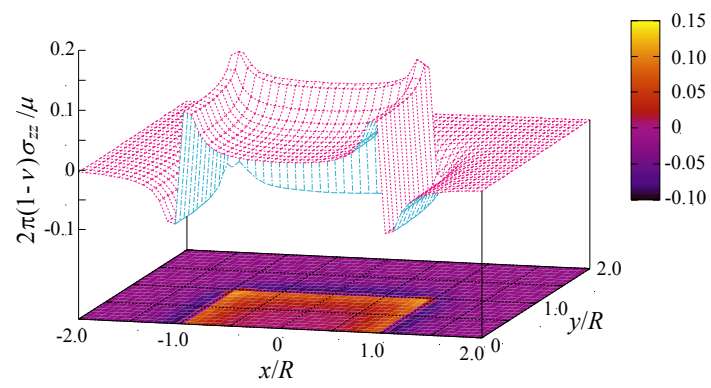

(b) Stress distribution of square loop on $x y$-plane

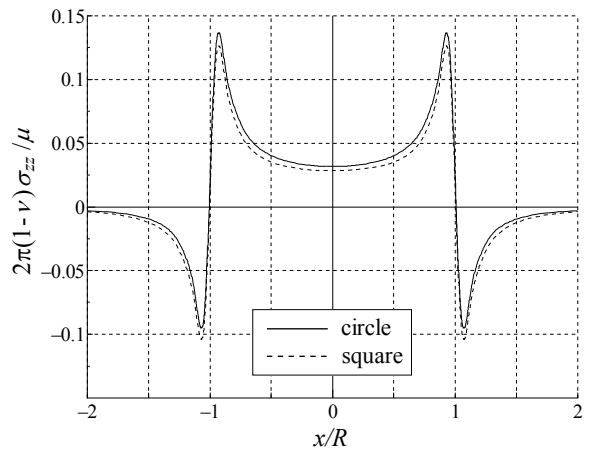

(c) Stress distribution of circular and square loop along $x$-axix

Fig. 4 Stress distribution of circular and square dislocation loops. 
work of classical theory. Here the dislocation loop is on the $z=0$ plane, with the normal stress component $\sigma_{z z}$ being presented. The stress distribution of a square loop can be evaluated using a dislocation mechanics simulation based on the vector formula of Devincre ${ }^{(17)}$, and a circular loop calculated with the analytic solution by de Wit ${ }^{(11)}$. Fig 4 (a) and (b) confirm that the distribution of a circular loop differs from that of a square loop. In Fig 4 (c), however, an analogous shape can be observed on the line across the center of the loop. Hence the dislocation energy can be approximated with relatively high accuracy as long as the entire length of the loops is first appropriately estimated.

As mentioned above, total dislocation energy is composed of the interaction energy, the self energy and the stacking fault energy. The contribution of each type to the total energy was then considered. A number of loops $N=50$ and loop radii $a$ of 15.5 and $10.0 \mathrm{~nm}$ for Al and $\mathrm{Cu}$, respectively, results in the energy contribution in terms of distance between loops given in Fig 5. The interaction energy dominates where the dislocations exist at short distances, as given in Fig 5 (a) and (b). Conversely the self energy is constant and dominates with increases in distance. The result is that interaction energy is of great significance with nanoindentation due to the high-density dislocations while the self energy is important with regard to the actual material.

\section{Relationship between burst load and burst width}

Atomistic simulations revealed that the stress distribution in nanoindentation with a spherical indenter is geometrically dependent on the radius of the indenter tip $r_{\mathrm{c}}{ }^{(6)}$. The relationship between the radius of PDL $a^{\prime}$ and contact radius at dislocation emission $r_{\mathrm{c}}$ obtained in atomistic simulations are given in Fig 6 (a). The radius $a$ ' was defined as the inscribed circle of the PDL (see Fig 6 (b)). It was discovered that a nearly linear relationship exists between $a^{\prime}$ and $r_{\mathrm{c}}$, and therefore the following least squares approximation could be used to estimate the inscribed circle at an arbitrary contact radius related to the dislocation emission.

$$
a^{\prime}=0.568 \times r_{c}+0.393 \quad[\mathrm{~nm}] .
$$

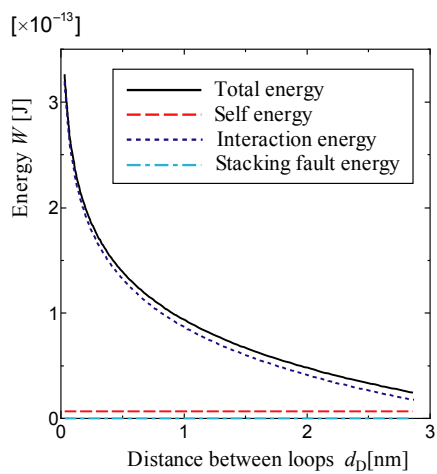

(a) $\mathrm{Al}\left(d_{\mathrm{D}}=0 \sim 3 \mathrm{~nm}\right)$

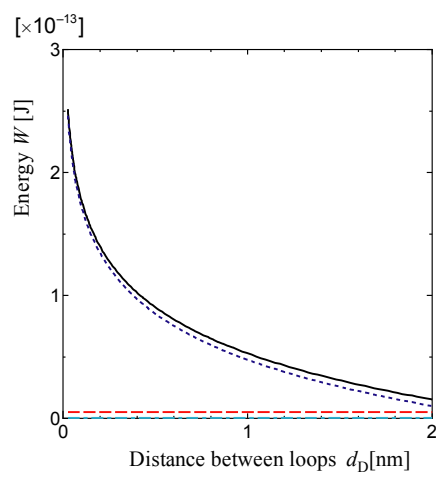

(c) $\mathrm{Cu}\left(d_{\mathrm{D}}=0 \sim 2 \mathrm{~nm}\right)$

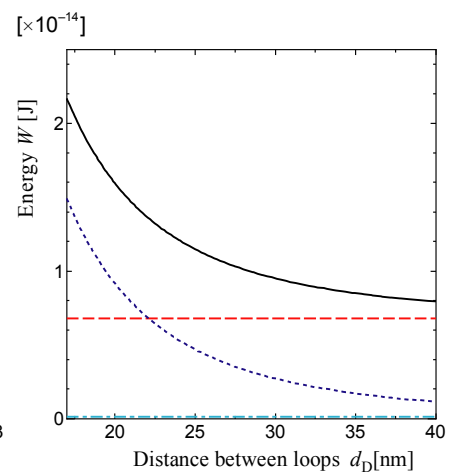

(b) $\mathrm{Al}\left(d_{\mathrm{D}}=17 \sim 40 \mathrm{~nm}\right)$

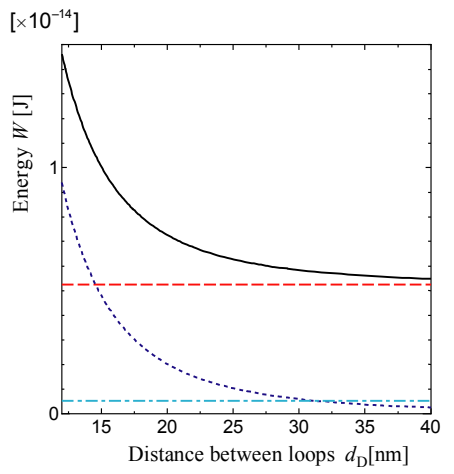

(d) $\mathrm{Cu}\left(d_{\mathrm{D}}=12 \sim 40 \mathrm{~nm}\right)$

Fig. 5 Relationship between contribution of dislocation energy and distance between loops. 


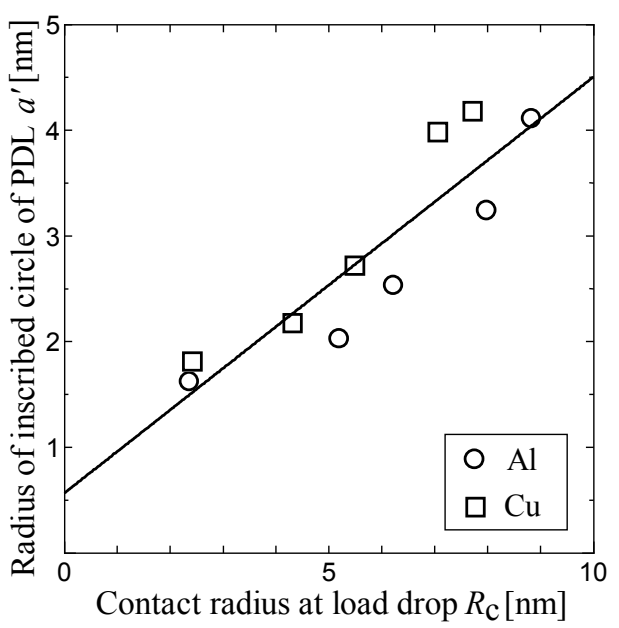

(a) Relationship between radius of PDL and contact radius

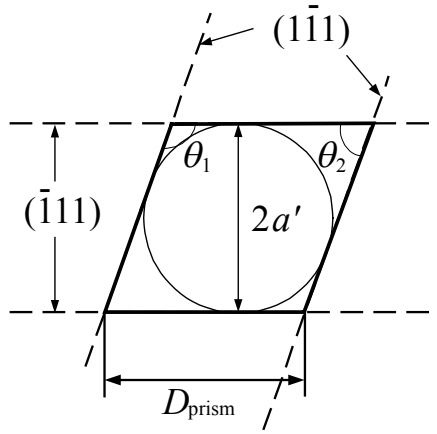

(b) Difinition of loop radius

Fig. 6 Relationship between loop radius and contact radius at the moment of dislocation emission.

A precondition given by Eq. (13) is imposed from a geometrical point of view with regard to the PDL as shown in Fig 6 (b). And as a result the total length of a PDL is then given with Eq. (14).

$$
\begin{aligned}
& a^{\prime}=\frac{\sqrt{2}}{3} D_{\text {prism }} . \\
& D_{\text {tot }}=4 D_{\text {prism }}=6 \sqrt{2} a^{\prime} .
\end{aligned}
$$

Using the above equations the equivalent length of a circular loop of the same length of a rhombic PDL can be obtained and thus directly reflected in classical theory evaluations.

We note again that the elastic strain energy stored by indentation is converted into the total dislocation energy, and assume that $W_{\mathrm{E}}=W_{\mathrm{D}}$. Preliminarily nanoindentation experiments were in good accord with the Hertzian solution with the radius of the indenter from 50 to $75 \mathrm{~nm}^{(3)}$, and variable constants of $R$ of 50 and $75 \mathrm{~nm}$, and the $d_{\mathrm{D}}$ of $1 b$ and $10 b$ were considered, respectively. The equation $W_{\mathrm{E}}=W_{\mathrm{D}}$ is solved and the relationship between burst width $h_{\mathrm{c}}$ and number of dislocations $N$ can be obtained. In addition, a dislocation makes the surface step corresponding to the Burgers vector of $z_{0}=b / \sqrt{2}$ in the case of the (001) indentation. And thus the burst width can be predicted with the number of dislocations as follows: $d_{\mathrm{B}}=N z_{0}$. The relationship between burst length and critical depth estimated using the proposed model for two different indenter radii and different distances between two loops is given in Fig 7. As a linear relationship exists between the number of

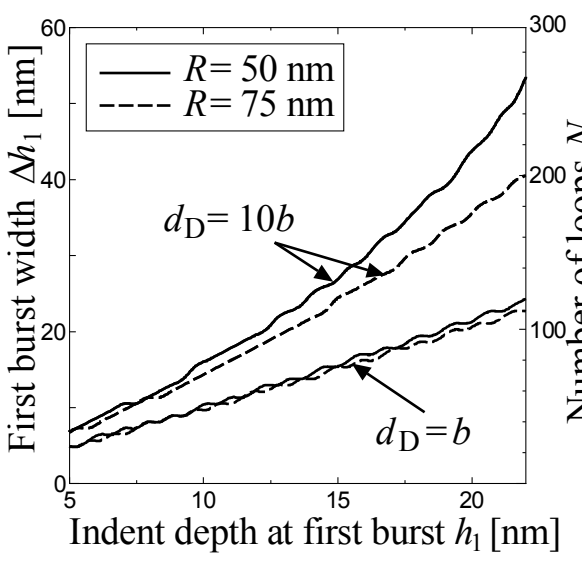

(a) $\mathrm{Al}$

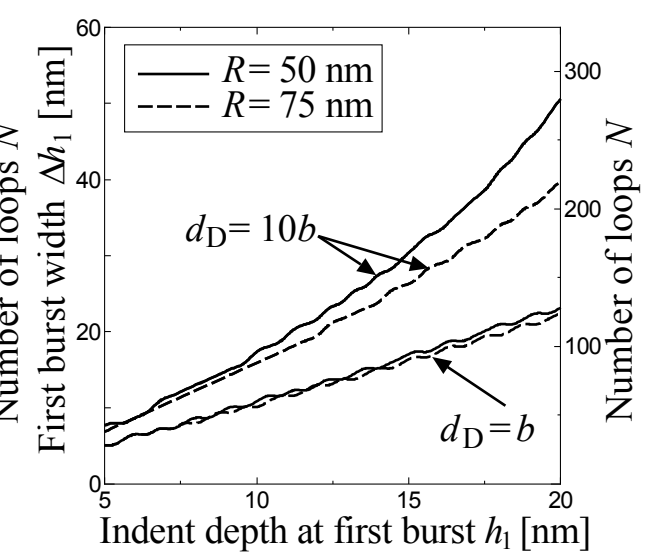

(b) $\mathrm{Cu}$

Fig. 7 Relationship between burst length and critical depth estimated on the assumption of two different radii of indenters and different distance between loops. 
dislocation loops and width with the first burst, the prediction can be made that more than 150 dislocation loops will be nucleated with bursts of a width of $50 \mathrm{~nm}^{(3)}$.

Finally the validity of the analytical model is verified through comparison with a nanoindentation experiment, with $\mathrm{Al}(001)$ nanoindentation being used as an example. The relationship between indent load at first burst $P_{1}$ and burst width $\Delta h_{1}$ as well as the result of our analytical model are given in Fig 8, where $P_{1}$ was directly calculated with $h_{1}$ in Fig 7 via the Hertzian solution. It confirmed that burst width increases with increasing indent load at the burst. This can be conceptually explained in that high elastic strain energy is stored with the increase in burst load and subsequently leads to a large amount of dislocation emissions. Atomistic simulations indicate that the burst load becomes higher when indented with large radii indenters ${ }^{(6)}$, thus leading to significant burst behavior being observed with larger radii indenters. The presence of bursts indicates that high-density dislocations are simultaneously nucleated in nanoindentation. And finally the discovery was made through both the strong consistency in Fig $8\left(R=50\right.$ and $\left.d_{\mathrm{D}}=10 b\right)$ and the relation to the number of dislocations in Fig 7, that the displacement burst is caused by surface steps corresponding to more than 150 simultaneously-emitted dislocation dipoles.

\section{Conclusions}

In the present paper displacement burst behavior caused by the collective dislocation nucleation observed in nanoindentation was discussed from the perspective of an analytical model of energy equilibrium based on Hertzian contact theory and classical dislocation theory. The interaction energy is of great significance with nanoindentation due to the high-density dislocations while the self energy is important with regard to the actual material. Our analytical model, both qualitatively and quantitatively, proved to be in good agreement with the experimental results, and consequently revealed that a displacement burst is induced from more than 150 simultaneously-emitted dislocation dipoles.

\section{Acknowledgement}

The authors would like to acknowledge the financial support of the Japan Society for the Promotion of Science (JSPS), Grant-in-Aid for JSPS Fellows (179504)(TT), Young Scientists (Start-up, 19860088)(TT), and Scientific Research (S, 20226004)(YS).

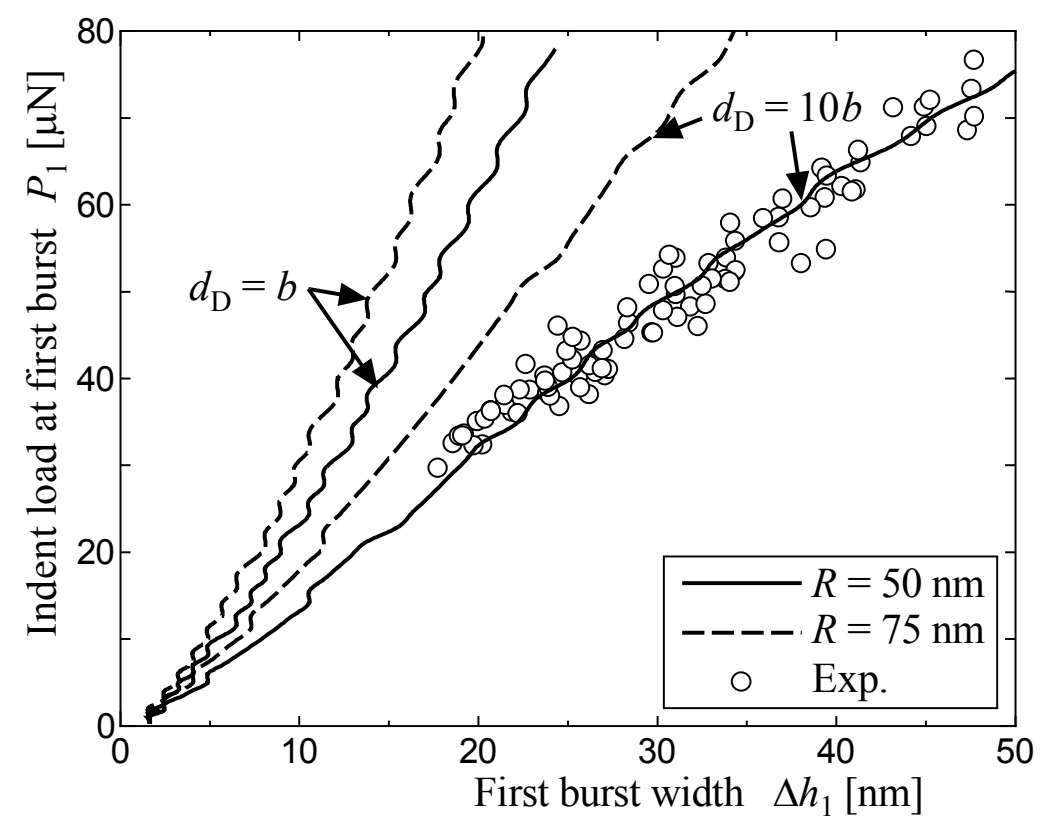

Fig. 8 Relationship between indent load at first displacement burst and burst width for Al. 


\section{References}

(1) Gerberich, W. W., Nelson, J. C., Lilleodden, E. T., Anderson, P. and Wyrobek, J. T., Indentation induced dislocation nucleation: The initial yield point, Acta Materialia, Vol. 44, No. 9 (1996), pp. 3585-3598.

(2) Suresh, S., Nieh, T.-G. and Choi, B. W., Nano-indentation of copper thin films on silicon substrates, Scripta Materialia, Vol. 41, No. 9 (1999), pp. 951-957.

(3) Shibutani, Y. and Koyama, A., Surface roughness effects on the displacement bursts observed in nanoindentation, Journal of Materials Research, Vol. 19, No. 1 (2004), pp. 183-188.

(4) Shibutani, Y., Tsuru, T. and Koyama, A., Nanoplastic deformation of nanoindentation: Crystallographic dependence of displacement bursts, Acta Materialia, Vol. 55, No. 5 (2007), pp. 1813-1822.

(5) Robertson, C. F. and Fivel, M. C., A Study of the submicron indent-induced plastic deformation, Journal of Materials Research, Vol. 14, No. 6 (1999), pp. 2251-2258.

(6) Tsuru, T. and Shibutani, Y., Atomistic simulations of elastic deformation and dislocation nucleation in $\mathrm{Al}$ under indentation-induced stress distribution, Modelling and Simulation in Materials Science and Engineering, Vol. 14, No. 5 (2006), pp. S55-S62.

(7) Tsuru, T. and Shibutani, Y., Anisotropic effects in elastic and incipient plastic deformation under (001), (110) and (111) nanoindentation in $\mathrm{Al}$ and $\mathrm{Cu}$, Physical Review B, Vol. 75, No. 3 (2007), pp. 035415.

(8) Johnson, K. L., Contacts Mechanics, Cambridge University Press, Cambridge, 2003 (First published 1985).

(9) Smithells, C. J., Smithells Metals Reference Book, 7th edition, Butterworth-Heinemann, London, 1992.

(10) Blin, J., Energie mutuelle de deux dislocations, Acta Metallurgica, Vol. 3 (1955), pp. 199-200.

(11) de Wit, R., The continuum theory of stationary dislocations, Solid State Physics (edited by Seitz, F. and Turnbull, D.), Vol. 10 (1960), pp. 249-292.

(12) Hirth, J. P. and Lothe, J., Theory of Dislocations, 2nd edition, Wiley, New York, 1982.

(13) Bullough, R. and Teukolsky, S. A., Elastic energy of phombus-shaped dislocation loop, Philosophical Magazine, Vol. 9, No. 98 (1964), pp. 315-329.

(14) Mishin, Y., Farkas, D., Mehl, M. J. and Papaconstantopoulos, D. A., Interatomic potentials for monoatomic metals from experimental data and ab initio calculations, Physical Review B, Vol. 59, No. 5 (1999), pp. 3393-3407.

(15) Mishin, Y., Mehl, M. J., Papaconstantopoulos, D. A., Voter, A. F. and Kress, J. D., Structural stability and lattice defects in copper: $\mathrm{Ab}$ initio, tight-binding, and embedded-atom calculations, Physical Review B, Vol. 63, No. 22 (2001), pp. 224106.

(16) Tsuru, T. and Shibutani, Y., Formation and critical shear stresses of prismatic dislocation loops observed around spherical precipitate in single crystalline aluminum and copper matrices (in Japanese), Transactions of the Japan Society of Mechanical Engineers, Series $A$, Vol. 71, No. 711 (2005), pp. 1445-1450.

(17) Devincre, B., Three dimensional stress field expressions for straight dislocation segment, Solid State Communications, Vol. 93, No. 11 (1995), pp. 875-878. 